\title{
Cámara Aérea para Medicamentos Presurizados
}

\author{
Dr. Carlos Aranda P. ${ }^{1}$; Klgo. Alan Power G ${ }^{2}$; Klgo. Ana María Merello M. ${ }^{3}$; \\ Klgo. Alejandro Parra V. ${ }^{3}$
}

\author{
Aerial Chamber for Administration of Aerosol of Salbutamol
}

\begin{abstract}
Seventeen children aged 5 to 11 years old, with exercise induced asthma (EIA) and an average exercise induced fall in their peak expiratory flow (PEF) of $27,5 \%$ were tested for prevention of EIA with Salbutamol in the aerosol form. The efficacy of the drug when the dosinteter was directiy applied into the mouth (standand method) was compared with its indirect application by means of an aerial chamber made of dischargeable plastic bottles for intravenous fluids, modified as to oclude both patient's mouth and nose with one of it's ends and to insert the dosimeter into the other one (alternetive method). The decrease in PEF fall was 96,6\%and 99,6\% with the standard and the alternative methods respectively; in both cases it was statistically significant $(p<0.011)$, suggesting the effectiveness of the alternative method and supporting our clinical observations in the treatment of wheezing infants. No side effects were observed.

(Key words: Aerial Chamber. Salbutamol Aerosol Administration. Exercise Induced Asthma. Prevention. Peak Expiratory Flow Fall Reduction).
\end{abstract}

El asma tiene alta prevalencia en nuestro país ${ }^{1,2}$. El uso de drogas broncodilatadoras por inhalación es el método más efectivo de tratamiento 4,5

La efectivjdad de la droga depende de la cantidad inhalada y de su penetración, que a su vez están determinadas por la tẻcnica de inhalación empleada $a^{6,7}$ y por las propiedades físicas del aerosol ${ }^{8,9,10}$.

La técnica de inhalación es particularmente importante en niños de corta edad ${ }^{11,22}$, en quienes la principal dificultad consiste en sincronizar la inhalación con la descarga, como ocurre con el empleo de dosificadores a presión ${ }^{13.14}$.

Varios autores han propuesto diferentes adaptaciones como bolsas colapsables ${ }^{15,16}$, tubos de extensión bucal ${ }^{12,17}$, aecocámaras ${ }^{13,19,19}$, las que no siempre resultan efectivas pues requieren un esfuerzo respiratorio exclusivamente bucal y además aumentan los costos.

Desde hace algún tiempo, en nuestro Servicio se está utilizando una mascatilla confeccionada con parte de un matraz de suero desechado, de aplicación buco-nasal, con el propósito de incor-

1. Médico del Servicio de Pediatría, Unidad Broncopulmonar, Hospital Félix Bulnes.

2. Jefe Unidad de Kinesiología, Hospital Félix Bulnes.

3. Unidad de Kinesiología, Hospital Félix Bulnes. porar a la población mencionada a este tipo de tratamiento, sin que sus resultados hayan sido comunicados.

La mascarilla que proponemos, constituye una verdadera cámara aérea para medicamentos envasados a presión (CAMP), que incluye boca $y$ nariz, y teóricamente disminuirían las dificultades de inhalación que presentan algunos pacientes.

Este estudio .se diseñó para evaluar este método alternativo, en comparación con el método clásico, midiendo la inhibición de la respuesta bronquial al ejercicio con drogas broncodilatadoras $($ Salbutamol $=$ Aerolin $\mathbf{R})$.

\section{MATERIAL Y METODOS}

El estudio se realizó con un grupo de 17 niños (entre 5 y 11 años de edad), que ingresaron a nuestro servicio de enfermedades broncopulmonares, por presentar antecedentes clinicos de asma inducida por el ejercicio (AIE), cuyas caracteristicas se presentan en la Tabla 1. En todos los pacientes se demostró AIE mediante una prueba estandar de ejercicio 20,21 , en la que tuvieron índices de caida (IC) ${ }^{22}$ del flujo respiratorio máximo (FEM), mayores o iguales que $12 \% 23$ e indices de labilidad bronquial (ILB) ${ }^{22}$ mayores o igual que $20 \% 24$.

Tres dias antes de las pruebas se suspendió el uso de todo tipo de drogas. Todos los niños 
Tabla 1.

Distribución según sexo, edad, talla y FEM basal de la muestra utilizada.

\begin{tabular}{|c|c|c|c|c|}
\hline Nin̄os & Sexo & Edad & $\begin{array}{l}\text { Talla } \\
(\mathrm{cm})\end{array}$ & $\begin{array}{l}\text { FEM } \\
\text { Basal }\end{array}$ \\
\hline 1 & masculino & 10 & 136.5 & 295 \\
\hline 2 & masculíno & 8 & 121.0 & 230 \\
\hline-3 & femenino & 7 & 120.0 & 290 \\
\hline 4 & femenino & 6 & 119.5 & 255 \\
\hline$m s$ & femenino & 10 & 140.0 & 365 \\
\hline *6 & femenino & 5 & 109.5 & 210 \\
\hline 7 & masculino & 8 & 131.0 & 320 \\
\hline * 8 & masculino & 7 & 121.0 & 290 \\
\hline 9 & masculino & 6 & 115.0 & 260 \\
\hline$* 10$ & masculino & 6 & 110.5 & 180 \\
\hline 11 & masculino & 5 & 105.0 & 160 \\
\hline 12 & femenino & 7 & 125.0 & 270 \\
\hline$* 13$ & masculino & 11 & 151.5 & 375 \\
\hline$\neq 14$ & femenino & 11 & 142.5 & 380 \\
\hline 15 & femenino & 9 & 132.0 & 310 \\
\hline 16 & masculino & 11 & 139.5 & 350 \\
\hline 17 & masculino & 5 & 101.1 & 175 \\
\hline$X$ & & 7.76 & 124.7 & 277 \\
\hline
\end{tabular}

- Se utilizó en primes término al método alternativo $y$ en segundo el clásico.

desconocian el uso de inhaladores. Se utilizó el flujómetro de Wright ${ }^{25}$ como instrumento de medida. El FEM de reposo en todas las pruebas estuvo dentro de limites normales.

Cada sujeto fue sometido a dos pruebas de inhibición del AIE con Salbutamol 200 ug $^{26}$, en 2 días consecutivos, utilizando un día el método clásico (MC) y al otro el método alternativo propuesto (MA), o viceversa. El MC, corresponde a las indicaciones habituales de uso, anexas al envase del medicamento. El MA consiste en la aplicación de una mascarilla hecha con parte de un matraz de $500 \mathrm{cc}$ de suero desechado, al que se le adaptaron ambos extremos, mediante perforación y recorte, a la boquilla del dosificador y a la cara del niño respectivamente (Figura 1), debiendo éste respirar por nariz y boca 5 ciclos naturales por cada descarga de aerosol desde el dosificador. La mascarilla o CAMP, fabricada de un material llamado "lapolén", cuya princjpal característica es ser hidrófobo, mide $7 \mathrm{~cm}$ de diámetro y $10 \mathrm{~cm}$ de longitud y tiene capacidad aproximada de $380 \mathrm{cc}$ (Figura 1 ).

La administración de la droga y las mediciones del thujo, fueron hechas por investigadores dife. rentes, ignorando, el último, el método usado, con el objeto de disminuir la influencia de factores subjetivos.

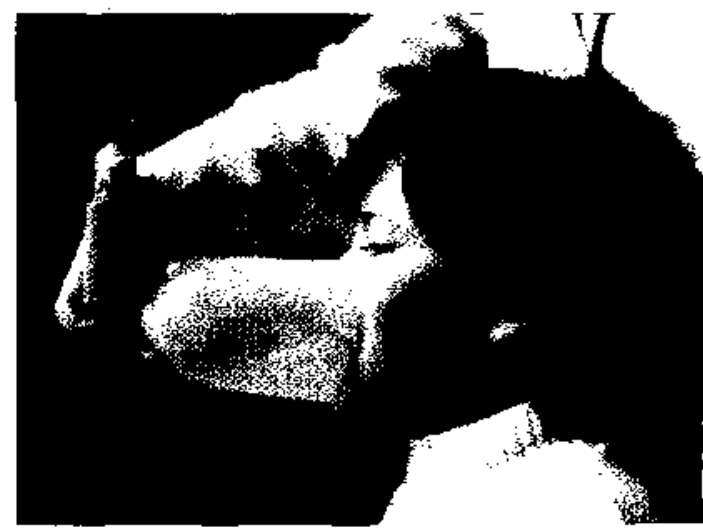

Figura 1: Fotografía en que se observa la aplicación de la mascarilla a la cara del niño y la forma de administrar el medicamento en aerosol.

Cada examen fue comparado mediante una prueba estadística paramétrica de análisis de datos pareados ( $T$ de Student $)^{27}$, para el IC y el ILB de cada examen. Se consideró el FEM de reposo pos-droga ( 5 min después de la inhalación de la droga), en vez de FEM de reposo pre-droga (inicial), sólo para los efectos de comparación entre las pruebas de bloqueo.

Se midió la frecuencia cardíaca antes y después de administrar la droga para cada método; también se buscó clínicamente la aparición de temblor en ambas manos como expresión de efectos indeseados del salbutamol.

Todos los exámenes se realizaron en el mismo lugar físico, en horario matinal y en tres dias consecutivos.

\section{RESULTADOS}

Las pruebas basales de ejercicio dierón ILB promedio $27,5 \%$ (D.E. $\pm 15,2$ ). Con la aplicación del método clásico el ILB promedio disminuyó a $12,7 \%$ (D.E. $\pm 7,5$ ) y el IC promedio a 3,4\% (D.E. \pm 7,9). Con el método alternativo se obtuvo un lLB promedio de $7,0 \%$ (D.E. $\pm 2,7$ ) y el lC bajó a $0,4 \%$ (D.E. $\pm 5,0)$. En otras palabras se produjo un bloqueo del IC de $96,6 \%$ con el MC y de $99,6 \%$ para el MA de administración. Ambos valores son estadísticamente significativos $(p<0,001)$ con respecto al examen basal.

Al comparar los resultados de ambos métodos entre si, no hubo diferencia estadísticamente significativa ( $p>0,05 y>0,01)$. (Figura $2 y$ Tabla 2).

En ningún paciente se observó temblor en las extremidades superiores. No hubo diferencias significativas en la frecuencia cardíaca al compa. 
Tabla 2.

Indices de labilidad bronquial y de caida del Flujo espiratorio máximo en las pruebas de ejercicio basai, y después de administrar aerosol de Salbutamol con dos métodos de inhalación.

\begin{tabular}{|c|c|c|c|c|c|c|}
\hline \multirow{2}{*}{ NIN̄OS } & \multicolumn{2}{|c|}{ PRUEBA BASAI } & \multicolumn{2}{|c|}{ METODO CLASICO } & \multicolumn{2}{|c|}{ METODO ALTERNATIVD } \\
\hline & I.L.B. & I.C. & I.L.B. & I.C. & 1.L.B. & $1 . C$. \\
\hline 1 & 27.1 & 22.0 & 12.3 & 6.2 & 9.4 & -3.1 \\
\hline 2 & 63.0 & 52.2 & 10.9 & -1.6 & 6.7 & -6.7 \\
\hline 3 & 24.1 & 20.7 & 6.9 & -3.5 & 4.8 & 1.6 \\
\hline 4 & 39.2 & 13.7 & 14.3 & 4.1 & 7.3 & 1.8 \\
\hline 5 & 37.0 & 30.1 & 8.5 & -7.0 & 7.7 & -3.8 \\
\hline 6 & 21.4 & 12.0 & 6.8 & -11.4 & 4.5 & -6.8 \\
\hline 7 & 25.0 & 15.6 & 23.3 & 11.7 & 6.8 & 0.0 \\
\hline 8 & 63.3 & 62.1 & 25.5 & 23.6 & 11.1 & 5.6 \\
\hline 9 & 36.5 & 28.9 & 7.5 & 1.9 & 5.8 & -3.8 \\
\hline 10 & 36.1 & 22.2 & 21.2 & 12.1 & 11.4 & 2.9 \\
\hline 11 & 50.0 & 12.5 & 29.3 & 2.4 & 7.0 & -7.0 \\
\hline 12 & 25.9 & 22.2 & 9.1 & 3.7 & 1.8 & 0.0 \\
\hline 13 & 32.0 & 30.7 & 10.0 & 4.3 & 4.1 & 5.5 \\
\hline 14 & 32.9 & 23.7 & 11.3 & 6.3 & 4.9 & 1.2 \\
\hline 15 & 30.7 & 30.7 & 8.8 & 3.5 & 11.9 & 10.2 \\
\hline 16 & 54.3 & 54.3 & 2.5 & 1.3 & 7.5 & 2.5 \\
\hline 17 & 28.6 & 14.3 & 8.3 & 0.0 & 5.9 & 5.9 \\
\hline$\overline{\mathrm{X}}$ & 36.7 & 27.5 & 12.7 & 3.4 & 7.0 & 0.4 \\
\hline $\mathrm{DE}$ & 12.8 & 15.2 & 7.5 & 7.9 & 2.7 & 5.0 \\
\hline
\end{tabular}

$p=$ no significativo.

I.L.B.: Indice Labilidad Bronqquiat.

I.C.: Indice de Caida del flujo espiratorio máximo.

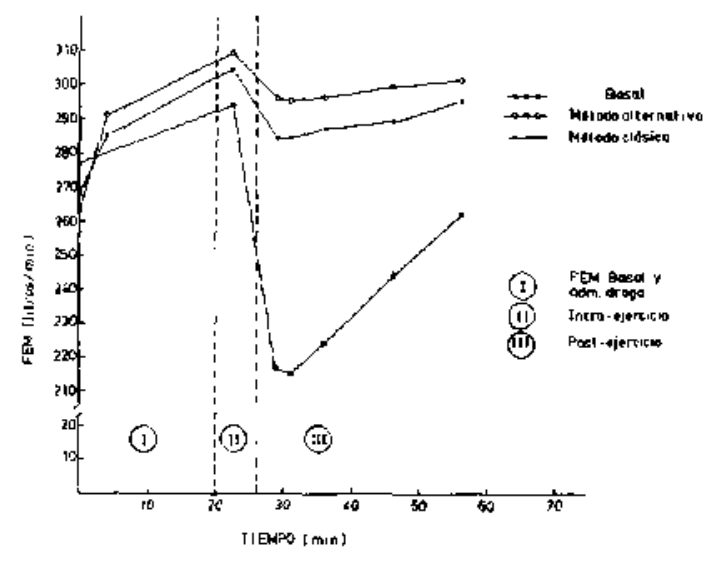

Figura 2: Comportamiento bronquial en la prueba de ejercicio basal $y$ en las pruebas de inhibición del AIE con los métodos alternativos y clásicos. Las Diferencias entre la prueba basal y ambas pruebas post-tratamiento con salbutamol son significativas $(p<0.01)$. La diferencia entre métodos clásico y alternativo no es significativa $(\mathrm{p}=0,05)$. rar los periodos pre y pos drogas $(p>0,01)$ de ambos métodos (Figura 3).

\section{COMENTARIOS}

Los resultados obtenidos nos permiten afirmar que el método altemativo propuesto es un recurso terapéutico eficaz para niños escolares, de muy fácil operación, disminuye el tiempo destinado a la instrucción del paciente, no requiere modificar su patrón ventilatorio $y$, por tratarse de un artefacto obtenido de material de desecho, su costo es casi nulo para nuestro medio.

Es importante recordar que aproximadamente el 10\% de cada dosis alcanza la vía respiratoria ${ }^{11}$, y que la mejor porción queda en la boca, en el orofaringe o es deglutida. Desconocemos si nuestro método modifica esta situación ya que a] incorporar los espacios nasales aumenta el área de impactación inercial, pero a su vez, la manten. 
एD tc. pre-droga fc. post-drogo

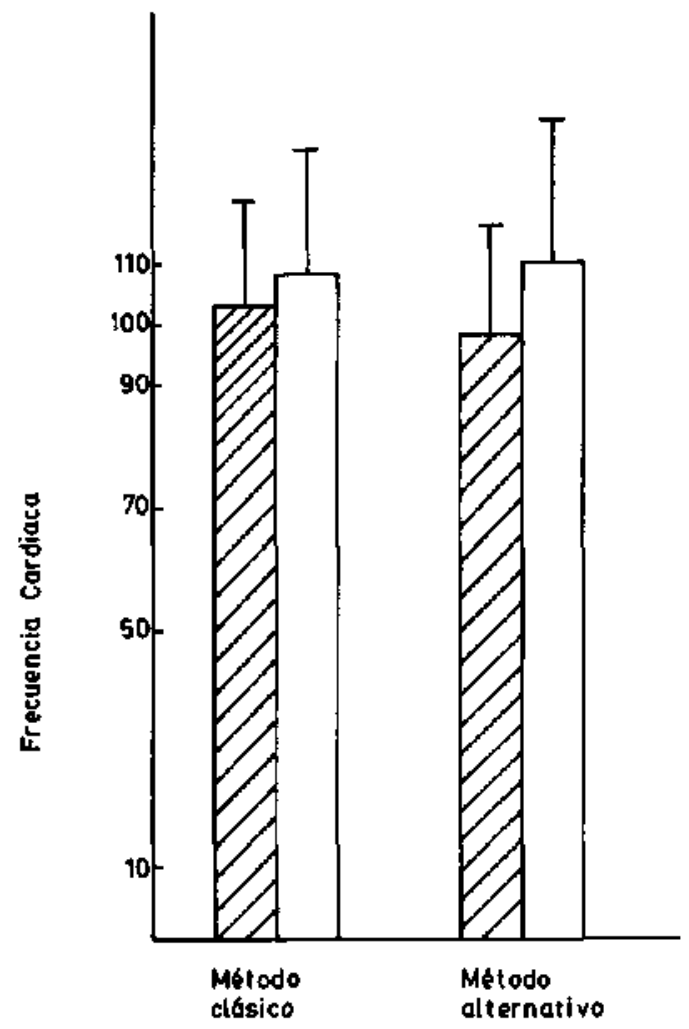

Fignra 3: Variaciones de la frecuencia cardiaca antes y después de la administración de salbutamol con dos métodos de administración.

ción del ciclo respiratorio espontáneo favorece la penetración y el depósito del aerosol ${ }^{28}$.

Estimamos que la técnica inhalatoria clásica presenta varios inconvenientes que impiden el mejor aprovechamiento de la droga inhalada. En efecto el procedimiento es iniciado con una espiración máxima, lo que produciria colapso de la via aérea pequefta, $y$ luego prosigue con una inspiración profunda, lo que determina una distribución irregular de partículas. Además, la cercanía de la boquilla a la lengua, las paredes bucales y la faringe, aumentaria la impactación inercjal, por la alta velocidad de flujo. A estos factores es necesario agregar la complejidad de las instrucciones y del método de aplicación que no siempre son bien comprendidos y aplicados por los pacientes.

La alternativa propuesta simplifica en gran medida las instrucciones, que en este caso son de adosar la mascarilla y abrir la boca, respirar normalmente y apretar el dosificador. Además la boquilla del inhalador está a $10 \mathrm{~cm}$ de la boca, lo que disminuiria la velocidad de las partículas, por la resistencia que opone el aire y la fuerza de gravedad, reduciendo la impactación inercial en la vía respiratoria superior. A su vez la mascarilla transparente, permite que el niño vea la acumulación del aerosol dentro de ella y observe su desaparición a medida que lo inhala.

En nuestro Servicio, por falta de drogas broncodilatadoras en solución para inhalación y escasez de sistemas de presión continua de gas, hemos usado la CAMP en lactantes $y$ preescolares, con obstrucción bronquial con resultados satisfactorios, en algunos de ellos, hemos comprobado mejoria en la $\mathrm{PO}_{2}$ transcutánea.

Los resultados obtenidos en nuestra investigación, respaldan esta experiencia clínica y de Laboratorio y sugiere que la CAMP puede ser útil en el tratamiento de la crisis obstructiva del lactante. En futuras investigaciones esperamos obtener información de manera más objetiva sobre esta posibilidad.

\section{RESUMEN}

Las dificultades para la inhalación de drogas envasadas a presión excluyen de esta importante arma terapéutica, a un numeroso grupo de pacientes. Buscando solucionar esto, desarrollamos un método alternativo consistente en una mascarilia confeccionada con parte de un matraz desechable para soluciones endovenosas.

El asma inducida por ejercicio fue inhibida por la inhalación de Salbutamol en dosificador presurizado administrado mediante el método clásico directo y el método alternativo usando la aerocámara descrita en un grupo de 17 niños asmáticos (5 a 11 años), con índices iniciales de caída del FEM iguales o mayores que $12 \%$, provocados con prueba de ejercicio estándar.

\section{REFERENCIAS}

1. Valenzuela P.: Prevalencia del asma bronquial en escolares de Stgo. Rev. Med. Chile. 109: 259, 1981.

2. Aranda C, Power A. y cols.: Determinación de la prevalencí de asma en escolares del Gran Stgo. Bol. Hosp. F. Bulnes C. 1: 13, 1984.

3. ibid. pp. 5.

4. Ancic P., Fuentes E. y Galleguillos E.: Estudio comparativo de broncodilatadores administrado por vía aerosol en pacientes asmáticos. Rev. Med. Chile. 109: 1157, 1981.

5. Pavio D. Thomson $M$. and Clarke S. Effect of lung function and mode of inhalation on penetration of aerosol into human lung. Thorax. 32: 194, 1977.

6. Dolovich M., Ruffing R. and Roberts R.: Optimal delivery of aerosol from metered dose inhalers. Chest. 80: 911, 1981. 
7. Newman S., Povio D. and Clorke S.: Improving the bronquial deposition of pressurized aerosols. Chest. B0: $909,1981$.

8. Newhouse $M$. and Ruffing R.: Deposition and fate of aerosolized drugs. Chest. 73: 936, 1978.

9. Morrow P.: Aerosol characterization and deposition, Am. Rev. Resp. Dis. 110: 88, 1974.

10. Task Group of Lung Dinamics: Deposition and retention models for internal dosimetry of the human respiratory tract. Health Physics. 12: 13, 1981.

11. Sistemas de inhalación de medicamentos para los asmóticas. Carta Médica. 19: 13, $198 \mathrm{~L}$.

12. Hodges $I$., Milner A. and Stokes G.: Assesment of a new device for delivering aerosols drugs to asthmatic chitdren. Arch. Dis Child. 56: 787, 1981.

13. Del Solar J.: Aerosoles broncodilatadores en la obstrucción bronquial. Apuntes Médicos Lab. Chile. 18: $17,1984$.

14. Grainger J.: Correct use of aerosols inhalers. C.M.A. Journal. 116: 584, 1977.

15. Tobin M., Jenouri G., Danta I. and cols.: Response to bronchodilator dug administration by a new reservoir aerosol delivery system and a review of other auxilary systems. Am. Rev. Resp. Dis 126: $670,1982$.

16. Lee $H$. y Evans $H$.: Administración a niños pequeños astnáticos de broncodilatadores en aerosol mediante una bolsa. Pediatrics (Ed. Esp.) 17: 140, 1984.

17. Ellul-Micallef R., Moren F. and cols.: Use of a special inhaler attachment in asthmatic children. Thorax. 35: 620, 1980 .

18. Freigang B.: New method of beclomethasone aerosol administration to children under 4 years. C.M.A.
Journal. 117: 130, 1977.

19. Newman $S$, Moren F., Pavio D. and cols.: Deposition of pressurized suspension gerosol inhaled through extension devices. Am. Rev, Resp. Dis. 124: 317, 1981.

20. Godfrey S., Silverman $M$. and Anderson S.: Proble$\mathrm{ms}$ of interpreting exercise-induced asthma. J. of All. and Clin. Immun. 52: 119, 1973.

21. Jiménez $J$., Cerutti E.: Broncoconstricción inducida pot ejercicio en nithos asmáticos y normales. Pediatría (Santiago). 24: 3, 1981.

22. Silverman $M$. and Anderson $S$.: Standarization of exercise test in asthmatic children. Arch. Dis Child. 47: 882,1972 .

23. Toussig $L$, Chernick $V_{*}$, Wood $R$. and Forrell $P$. Standarization lung function testing in chidren. $J$. Pediatr. 97: 668, 1180.

24. Diaz P., Ferninalez S. y Spratia A.: Estudio del indice de labilidad bronquial producido con el test de ejetcicio en niños asmáticos y normales. Tesis para optar al título de Kinesiólogo de la Unwersidad del Norte. pp. 69, 1982.

25. Wright B. and MC Kerrow C: Maximun forced expiratory flow rate as a measure of ventilatory capacity, with a description of a new portable inštrument for measurìg it. Brit. Med. J. 2: 1041 , 1959.

26. Godfrey $S$. and Koning P.: Inhibition of exerciseinduced asthma by different pharmacological pathways. Thorax. 31: 137, 1977.

27. Schefler W.: Hioestadística. 2a. Ed. Fondo Educativo Interamericano. S.A. México. pp. 94, 1981.

28. López $R$.: Insuficiencia Respiratoria. 2a. Ed. Editorial Jims. Barcelona. pp. 156, 1975. 